\title{
Curcumin inhibits superoxide dismutase-induced epithelial-to-mesenchymal transition via the PI3K/Akt/NF-кB pathway in pancreatic cancer cells
}

\author{
WEI LI $^{1}$, ZHENGDONG JIANG $^{1}$, XUE XIAO $^{2}$, ZHENG WANG $^{1}$, ZHENG WU $^{1}$, QINGYONG MA ${ }^{1}$ and LEI CAO ${ }^{2}$ \\ ${ }^{1}$ Department of Hepatobiliary Surgery, The First Affiliated Hospital of Xi'an Jiaotong University; \\ ${ }^{2}$ Department of Pharmacology, School of Basic Medical Sciences, \\ Xi'an Jiaotong University Health Science Center, Xi'an, Shaanxi 710061, P.R. China
}

Received October 22, 2017; Accepted February 21, 2018

DOI: 10.3892/ijo.2018.4295

\begin{abstract}
Curcumin is a natural polyphenol compound derived from turmeric. It possesses multiple pharmacological properties, including antioxidant, anti-inflammatory and antitumor progression properties. Our recent study demonstrated that superoxide dismutase (SOD)-dependent production of hydrogen peroxide $\left(\mathrm{H}_{2} \mathrm{O}_{2}\right)$ promoted the invasive and migratory activity of pancreatic cancer cells. However, whether curcumin suppresses SOD-induced cancer progression and the related mechanisms remains unclear. Since epithelial-tomesenchymal transition (EMT) plays a key role in tumor metastasis, the aim of the present study was to examine whether curcumin intervenes with SOD-induced EMT in pancreatic cancer and the underlying mechanism. The human pancreatic cancer cells BxPC-3 and Panc- 1 were exposed to SOD in the presence or absence of curcumin, catalase (CAT, a scavenger of $\mathrm{H}_{2} \mathrm{O}_{2}$ ), or LY 294002 [a phosphoinositide-3 kinase (PI3K) inhibitor]. Intracellular reactive oxygen species (ROS) and $\mathrm{H}_{2} \mathrm{O}_{2}$ were evaluated by 2,7-dichlorodihydrofluorecein diacetate and $\mathrm{H}_{2} \mathrm{O}_{2}$ assay, respectively. The activation of p-Akt and p-nuclear factor (NF)- $\kappa \mathrm{B}$ were examined by western blotting. The migratory and invasive abilities of pancreatic cancer cells were tested by the wound healing and Transwell invasion assays. The expression of E-cadherin, $\mathrm{N}$-cadherin and vimentin (EMT-related genes) were measured by reverse transcription-quantitative polymerase chain reaction and western blotting at the mRNA and protein levels, respectively. The findings of the present study demonstrated
\end{abstract}

Correspondence to: Dr Lei Cao, Department of Pharmacology, School of Basic Medical Sciences, Xi'an Jiaotong University Health Science Center, 76 West Yanta Road, Xi'an, Shaanxi 710061, P.R. China E-mail: 289421999@qq.com; leicao@mail.xjtu.edu.cn

Key words: curcumin, superoxide dismutase, phosphoinositide 3 kinase/Akt pathway, epithelial-to-mesenchymal transition, nuclear factor- $\kappa \mathrm{B}$ pathway, pancreatic cancer that curcumin decreased SOD-induced production of ROS and $\mathrm{H}_{2} \mathrm{O}_{2}$ in BxPC-3 and Panc-1 cells. Curcumin was able to suppress SOD-induced invasion and migration, and it also regulated the expression of the above-mentioned EMT-related genes and cell morphology. SOD-induced cell invasion was also inhibited by catalase and LY 294002. Furthermore, the levels of p-Akt and p-NF- $\kappa$ B caused by SOD could be offset by treatment with curcumin and LY 294002. To summarize, these results demonstrated that curcumin was able to prevent SOD-driven $\mathrm{H}_{2} \mathrm{O}_{2}$-induced pancreatic cancer metastasis by blocking the PI3K/Akt/NF- $\mathrm{KB}$ signaling pathway. The use of curcumin to inhibit the $\mathrm{H}_{2} \mathrm{O}_{2} / \mathrm{Akt} / \mathrm{NF}-\mathrm{\kappa B}$ axis may be a promising therapeutic approach to the treatment of patients with pancreatic cancer.

\section{Introduction}

Pancreatic cancer is a highly aggressive and lethal human malignancy, with a very low 5-year survival rate worldwide $(8 \%)$. The low survival rate is partly attributed to the fact that over half of the cases are diagnosed at an advanced stage, for which the 5-year survival may be as low as $3 \%$ (1). An estimated 53,670 new pancreatic cancer cases were diagnosed in 2017, which may result in 43,090 cancer-related deaths in the United States (1). In China, pancreatic cancer is the seventh most fatal disease, with an even lower 5-year survival rate $(4.1 \%)(2)$. The routine treatments include surgery, radiation and chemotherapy (with gemcitabine, 5 -fluorouracil or their combination). However, $\sim 80 \%$ of pancreatic cancer subjects are diagnosed when the tumor is unresectable and/or has developed distant metastasis, and acquired resistance to chemotherapeutic treatment always develops during the course of the disease (3). Therefore, more comprehensive and effective interventions are urgently required to improve the treatment outcome of pancreatic cancer patients.

Curcumin is a natural polyphenol compound derived from turmeric. It possesses several biological properties, including anti-inflammatory and anti-oxidant properties, and also plays a key role in inhibiting the initiation, progression and metastasis of several tumors (4). Curcumin exerts its anticancer effects by targeting multiple intracellular signaling pathways, 
including mitogen-activated protein kinase (MAPK), nuclear factor (NF)- $\mathrm{KB}$, Akt and Wnt/3-catenin, among others (5-7). In addition, we recently demonstrated that curcumin inhibited hypoxia-induced epithelial-to-mesenchymal transition (EMT) in pancreatic cancer cells via blockade of the hedgehog signaling pathway (8).

EMT has been recognized not only as a physiological mechanism in mammalian embryonic development and tissue remodeling, but also as an important phenomenon observed in tumorigenesis and cancer development (9). During EMT, epithelial cells lose their polarity, cell-cell tight junctions and adhesive connections, and acquire mesenchymal characteristics (10). The hallmark of EMT is the loss of E-cadherin expression (an epithelial marker) and the gain of vimentin and $\mathrm{N}$-cadherin expression (mesenchymal markers) (10). We previously demonstrated that several factors could induce EMT in pancreatic cancer cells, including hypoxic (8) and hyperglycemic environment (11), as well as the induction of superoxide dismutase (SOD) (12).

Reactive oxygen species (ROS), including hydrogen peroxide $\left(\mathrm{H}_{2} \mathrm{O}_{2}\right)$, are a group of chemically reactive molecules derived from oxygen. Intrinsic antioxidant enzymes play a crucial role in the regulation of oxidative stress in cells. SOD is one of the primary cellular antioxidants, and it can catalyze the conversion of superoxide anion to $\mathrm{H}_{2} \mathrm{O}_{2}$, which is cleared by catalase (CAT) (13). Due to the cytoprotective effects of SOD, its overexpression has been associated with increased incidence of tumor metastasis (14). In our previous study, we verified that the invasive ability and EMT of pancreatic cancer cells was aggravated by SOD-dependent production of ROS via the extracellular signal-regulated kinase (ERK)/NF- $\mathrm{KB}$ signaling pathway (12). However, whether curcumin can repress the SOD-induced progression of pancreatic cancer, and the possible underlying mechanisms, have not been fully elucidated.

The aim of the present study was to verify our hypothesis and determine whether curcumin has the potential to suppress SOD-driven $\mathrm{H}_{2} \mathrm{O}_{2}$-induced invasive and migratory abilities and inhibit EMT in pancreatic cancer cells. We also aimed to examine the effect of curcumin on SOD-induced activation of the phosphoinositide-3 kinase (PI3K)/Akt signaling pathway and the transcription factor NF- $\kappa \mathrm{B}$, to determine whether using curcumin to inhibit the $\mathrm{H}_{2} \mathrm{O}_{2} / \mathrm{Akt} / \mathrm{NF}-\kappa \mathrm{B}$ axis may be a promising approach to the treatment of pancreatic carcinoma.

\section{Materials and methods}

Cell culture and reagents. The human pancreatic cancer cell lines BxPC-3 and Panc-1 were purchased from the American Type Culture Collection (Manassas, VA, USA). The cells were cultured in Dulbecco's modified Eagle's medium (DMEM), which contains $10 \%$ dialyzed heat-inactivated fetal bovine serum (FBS), $100 \mathrm{U} / \mathrm{ml}$ penicillin and $100 \mu \mathrm{g} / \mathrm{ml}$ streptomycin in a humidified atmosphere of $5 \% \mathrm{CO}_{2}$ at $37^{\circ} \mathrm{C}$. The cells exponentially grew in complete medium and treated with $400 \mathrm{U} /$ $\mathrm{ml} \mathrm{SOD}$, with or without $20 \mu \mathrm{M}$ curcumin, $400 \mathrm{U} / \mathrm{ml} \mathrm{CAT}$ or $10 \mu \mathrm{M}$ LY 294002 (a PI3K inhibitor) for the indicated time intervals, according to the experimental protocol. DMEM and FBS were purchased from Gibco; Thermo Fisher Scientific (Grand Island, NY, USA). Curcumin, CuZnSOD, CAT and
LY294002 were purchased from Sigma-Aldrich; Merck KGaA (St. Louis, MO, USA). The hydrogen peroxide assay kit and the ROS assay kit were obtained from Beyotime (Jinan, China). Millicell culture plate inserts were purchased from Millipore (Bedford, MA, USA). Matrigel was purchased from BD Biosciences (Bedford, MA, USA). Primary antibodies [dilution 1:100 in phosphate-buffered saline (PBS)-Tween-20] against E-cadherin (sc-52328), N-cadherin (sc-53488) and vimentin (sc-66002) were obtained from Santa Cruz Biotechnology (Santa Cruz, CA, USA). The anti-Akt (no. 9272), anti-phospho-Akt (anti-p-Akt, Ser473, no. 4060), anti-NF-kB

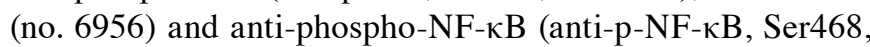
no. 3039) antibodies (dilution 1:200 in PBS-Tween-20) were obtained from Cell Signaling Technology (Beverly, MA, USA). Other reagents were purchased from common commercial sources. All drug solutions were freshly prepared on the day of testing.

Measurement of intracellular ROS. The level of intracellular ROS was examined using the ROS assay kit according to the manufacturer's instructions. Briefly, pancreatic cancer cells were incubated with 2,7-dichlorodihydrofluorescein diacetate (DCFDA) for $30 \mathrm{~min}$. After washing 3 times, fluorescence intensity was measured using a fluorometer (Becton-Dickinson, Franklin Lakes, NJ, USA) with excitation at $488 \mathrm{~nm}$ and emission at $525 \mathrm{~nm}$.

Hydrogen peroxide assay. The level of intracellular $\mathrm{H}_{2} \mathrm{O}_{2}$ was tested using a hydrogen peroxide assay kit. In this kit, $\mathrm{H}_{2} \mathrm{O}_{2}$ oxidizes ferrous $\left(\mathrm{Fe}^{2+}\right)$ to ferric $\left(\mathrm{Fe}^{3+}\right)$ ions; then, $\mathrm{Fe}^{3+}$ ions combine with the indicator dye xylenol orange to form a complex and produce a visible purple-colored complex, which may be measured using a microplate reader at a wavelength of 560-590 nm (Bio-Rad Laboratories, Inc., Hercules, CA, USA).

Transwell Matrigel invasion assay. The 8.0- $\mu \mathrm{m}$ pore inserts were covered with $25 \mu \mathrm{l}$ Matrigel. BxPC-3 and Panc-1 cells were subjected to $24 \mathrm{~h}$ serum starvation and were then suspended in DMEM supplemented with $1 \%$ FBS in the upper chamber at a density of $5 \times 10^{4}$, with or without SOD, SOD with curcumin, CAT or LY 294002. In addition, $500 \mathrm{ml}$ DMEM with 20\% FBS were placed in the lower chamber. The Matrigel invasion chamber was placed in a humidified tissue incubator for $48 \mathrm{~h}$. The non-invading cells were cleared away from the top surface using a cotton swab and the filter was rinsed with phosphate-buffered saline, fixed and stained with crystal violet. Finally, the stained cells on the bottom surface were counted to evaluate the invasive capacity of cancer cells. Three random fields were captured at a magnification of $x 20(n=3)$.

Wound healing assay. Cells were seeded in 24-well plates $\left(1.0 \times 10^{5}\right.$ cells $\left./ 500 \mu \mathrm{l}\right)$. After the cells had grown to a $90-100 \%$ confluence, a sterile pipette tip was used to create a scratch wound. Cellular debris was cleared away and the remaining cells were allowed to grow and migrate in the plate for $24 \mathrm{~h}$. Images were captured at time points 0 and $24 \mathrm{~h}$ post-wounding by a Nikon Diaphot TMD inverted microscope (magnification, $\mathrm{x} 10$ ). The relative distance traveled by the leading edge from 0 to $24 \mathrm{~h}$ was assessed using Photoshop software $(\mathrm{n}=5)$. 

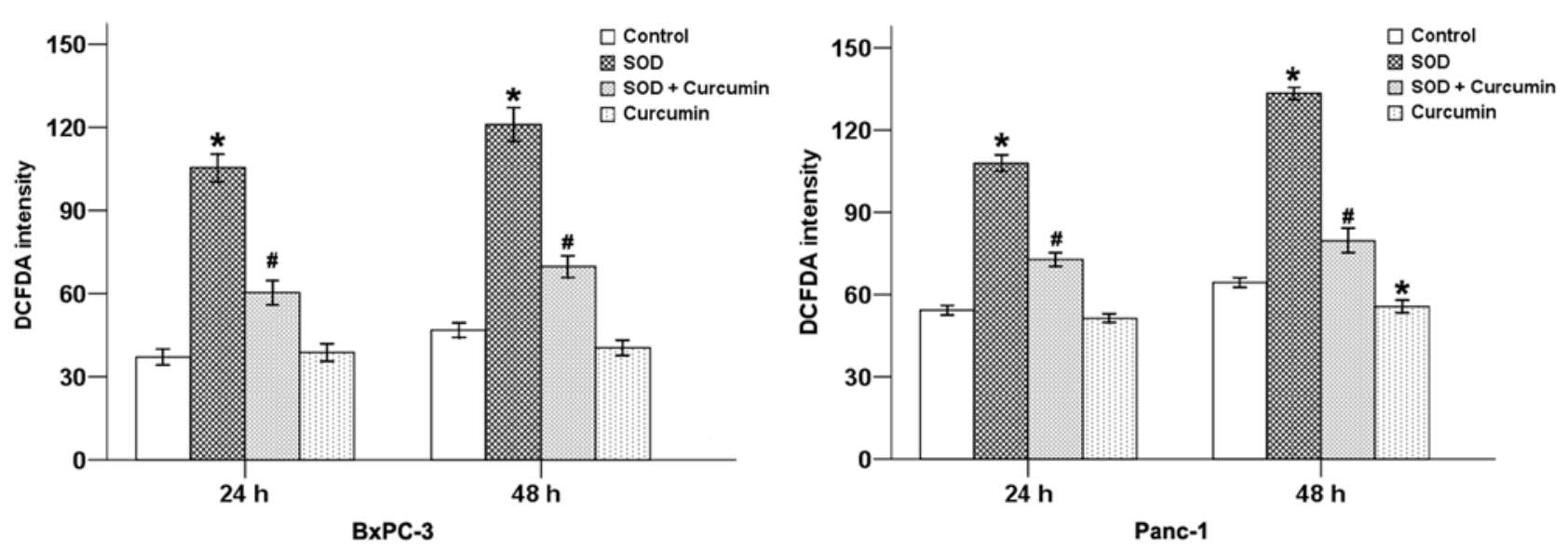

Figure 1. Effects of curcumin on SOD-induced ROS production. BxPC-3 and Panc-1 cells were treated with $20 \mu \mathrm{M}$ curcumin for 24 or $48 \mathrm{~h}$ following SOD $(400 \mathrm{U} / \mathrm{ml})$ administration to detect the level of ROS. ${ }^{\prime \prime} \mathrm{P}<0.05$ vs. control group; ${ }^{\prime} \mathrm{P}<0.05$ vs. SOD group. SOD, superoxide dismutase; ROS, reactive oxygen species; DCFDA, 2,7-dichlorodihydrofluorescein diacetate.

Reverse transcription-quantitative polymerase chain reaction $(R T-q P C R)$. Total RNA was extracted from cancer cells using the Fastgen200 RNA isolation system (Fastgen, Shanghai, China). cDNA was reverse-transcribed from RNA using the Fermentas RevertAid $^{\mathrm{TM}}$ Kit (MBI Fermentas, Burlington, ON, Canada). The primer sequences were as follows: E-cadherin, F: 5'-ATT CTGATTCTGCTGCTCTTG-3' and R: 5'-AGTCCTGGTCCTC TTCTCC-3'; N-cadherin, F: 5'-TGTTTGACTATGAAGGCAG TGG-3' and R: 5'-TCAGTCATCACCTCCACCAT-3'; vimentin, F: 5'-AATGACCGCTTCGCCAAC-3' and R: 5'-CCGCATCTCC TCCTCGTAG-3'; and $\beta$-actin,F: 5'-GACTTAGTTGCGTTACAC CCTTTCT-3' and R: 5'-GAACGGTGAAGGT GACAGCAGT-3'.

The PCR conditions were as follows: $30 \mathrm{sec}$ at $95^{\circ} \mathrm{C}$, followed by 40 cycles at $95^{\circ} \mathrm{C}$ for $5 \mathrm{sec}, 60^{\circ} \mathrm{C}$ for $30 \mathrm{sec}$ and $72^{\circ} \mathrm{C}$ for $30 \mathrm{sec}$. A dissociation curve analysis was applied after each RT-qPCR experiment. The relative gene expression was calculated using the previously described $2^{-\Delta \Delta C q}$ method (15).

Western blotting. Identical amounts of protein from cancer cells were loaded on a denaturing sodium dodecyl sulfatepolyacrylamide gel. After gel electrophoresis, the protein was transferred onto nitrocellulose membranes. Subsequently, the membranes were initially blocked with $5 \%$ non-fat dry milk in Tris-buffered saline (TBS) for $2 \mathrm{~h}$, followed by incubation with different primary antibodies (E-cadherin, $\mathrm{N}$-cadherin, vimentin, Akt, p-Akt, NF- $\kappa B$, p-NF- $\kappa B$ and $\beta$-actin antibodies) at $4^{\circ} \mathrm{C}$ overnight. The membranes were then incubated with secondary goat anti-mouse or goat anti-rabbit antibodies (Sigma-Aldrich; Merck $\mathrm{KGaA}$ ) for $2 \mathrm{~h}$ at room temperature. Immunopositive bands were developed using an enhanced chemiluminescence (ECL) detection system (Amersham, Piscataway, NJ, USA). All analyses were conducted in triplicate.

Statistical analysis. Statistical analysis was performed using SPSS software, version 17.0 (SPSS Inc., Chicago, IL, USA). Data are presented as the means \pm standard error of the mean of three replicate assays. Differences between the groups were analyzed by analysis of variance with Dunnett's post-hoc test. Statistical significance was set at $\mathrm{P}<0.05$. All experiments were repeated independently at least three times.

\section{Results}

Curcumin inhibits SOD-induced oxidative stress in pancreatic cancer cells. ROS caused by oxidative stress damage cellular DNA, proteins and lipids, and produce toxic and highly mutagenic metabolites that may modify tumor behavior (16). We previously demonstrated that SOD stimulated the production of $\mathrm{H}_{2} \mathrm{O}_{2}$ in pancreatic cancer cells (12). As curcumin possess antioxidant properties, in the present study, we first examined the effects of curcumin on the production of $\mathrm{ROS}$ and $\mathrm{H}_{2} \mathrm{O}_{2}$ in BxPC-3 and Panc-1 cells following treatment with SOD. Our results revealed that SOD significantly increased the DCFDA staining intensity (Fig. 1) and the level of $\mathrm{H}_{2} \mathrm{O}_{2}$ (Fig. 2) in pancreatic cancer cells, while curcumin was able to counterbalance these effects at 24 and $48 \mathrm{~h}$ in both cancer cell types.

Curcumin downregulates the activation of the Akt/NF- $\kappa B$ signaling pathway. The PI3K/Akt pathway plays an important role in numerous cellular processes, such as cell metabolism, survival, differentiation, proliferation, motility and angiogenesis (17). It has been proven that cancer cell signaling is mediated by PI3K/Akt via activation of the transcription factor $\mathrm{NF}-\kappa \mathrm{B}$, which is associated with cell proliferation, migration and invasion (18).

We previously demonstrated that SOD promotes activation of the ERK/NF- $\kappa \mathrm{B}$ signaling pathway (12). The findings of the present study indicated that SOD could also upregulate the $\mathrm{Akt} / \mathrm{NF}-\mathrm{\kappa B}$ signaling pathway, as the phosphorylated levels of both Akt and NF- $\kappa B$ genes were found to be significantly elevated following SOD addition (Fig. 3A). When SOD was added to the cell in culture medium along with curcumin, the expression of p-Akt and p-NF- $\mathrm{kB}$ was strongly downregulated (Fig. 3A). Our results also demonstrated that curcumin alone was able to inhibit the expression of p-AKT and p-NF-kB (Fig. 3B). In addition, LY 294002, a PI3K inhibitor, also suppressed the expression of both p-Akt and p-NF- $\mathrm{KB}$, indicating that NF- $\mathrm{KB}$ is located downstream of the PI3K/Akt pathway (Fig. 3C).

Curcumin inhibits SOD-induced invasion of pancreatic cancer cells. Malignant tumors are often characterized by metastasis, which is dissemination of cells from a primary 

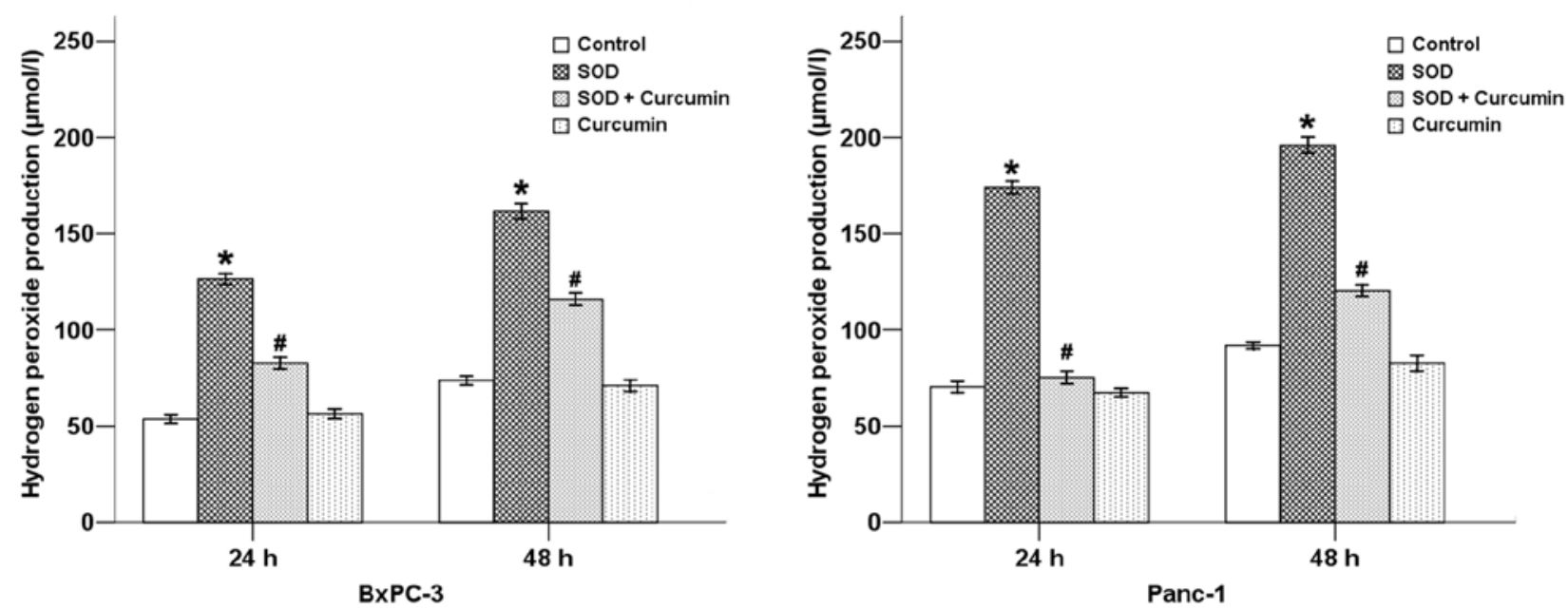

Figure 2. Effects of curcumin on SOD-induced $\mathrm{H}_{2} \mathrm{O}_{2}$ production. Pancreatic cancer cells were treated with $20 \mu \mathrm{M}$ curcumin for 24 or $48 \mathrm{~h}$ following SOD ( $400 \mathrm{U} / \mathrm{ml}$ ) administration to detect the production of $\mathrm{H}_{2} \mathrm{O}_{2}$. $\mathrm{P}<0.05$ vs. control group; ${ }^{*} \mathrm{P}<0.05$ vs. SOD group. SOD, superoxide dismutase.

site to colonize distant organs. The ability of cancer cells to migrate and invade other tissues are two important steps in the development of metastasis. To verify whether curcumin is able to affect SOD-induced cell invasion in pancreatic cancer cells, a Transwell invasion assay was performed. As shown in Fig. 4, the mean number of cells invading into the lower chamber increased in the presence of SOD after a 48-h incubation, and this increase was inhibited by co-treatment with curcumin. In addition, CAT and LY 294002 also suppressed the effect of SOD, indicating that SOD-induced invasion is associated with the $\mathrm{H}_{2} \mathrm{O}_{2}$ /Akt axis (Fig. 4).

Curcumin suppresses SOD-induced migration of pancreatic cancer cells. A classic wound healing assay was next conducted to assess the effect of curcumin on SOD-induced cell motility in pancreatic cancer cells. Our results revealed that the migration of cancer cells was significantly promoted by SOD after a 24-h incubation. Delayed wound closure was observed following treatment with curcumin in the two cancer cell types. The increase in cell migration was also inhibited by co-treatment with CAT and LY 294002. Therefore, curcumin likely exerts its inhibitory effects on cancer cell motility through suppression of the $\mathrm{H}_{2} \mathrm{O}_{2} / \mathrm{Akt} / \mathrm{NF}-\kappa \mathrm{B}$ axis (Fig. 5).

Curcumin inhibits SOD-promoted EMT in pancreatic cancer cells. EMT is considered a prerequisite for cells to adopt a motile and invasive phenotype and eventually become metastatic. EMT includes four important steps: Loss of epithelial cell adhesion, expression of mesenchymal proteins and acquisition of a mesenchymal-like phenotype, degradation of the basement membrane, and enhanced cell migration and invasion, which facilitate tumor cell invasion into the stroma and entrance into the circulation (10). Our previous study demonstrated that SOD-induced $\mathrm{H}_{2} \mathrm{O}_{2}$ production is able to promote EMT in pancreatic cancer cells, resulting in increased cell motility and invasion via activation of the ERK signaling pathway (12). In the present study, we demonstrated that cancer cell morphology changed from a typical epithelial phenotype to a mesenchymal phenotype following SOD treatment for $48 \mathrm{~h}$, which was counterbalanced by curcumin (Fig. 6A).
A hallmark of EMT includes a marked decline in E-cadherin expression (a cell-cell adhesion molecule) and increase in vimentin and $\mathrm{N}$-cadherin expression (mesenchymal markers) (11). To further verify the effect of curcumin on SOD-induced EMT, the mRNA and protein expression levels of several EMT-related factors (E-cadherin, N-cadherin and vimentin) were assessed after the cells were treated with SOD in the presence or absence of curcumin. As shown in Fig. 6B, SOD decreased the mRNA level of E-cadherin and increased the mRNA level of $\mathrm{N}$-cadherin and vimentin, whereas curcumin significantly reversed these effects. As shown in Fig. 6C, SOD exposure could modulate the protein levels of EMT-related factors, while curcumin suppressed these effects of SOD.

To summarize the abovementioned results, SOD was able to induce EMT progression and facilitate tumor invasion and migration via the production of $\mathrm{H}_{2} \mathrm{O}_{2}$ in BxPC-3 and Panc-1 pancreatic cancer cells, whereas these effects were counterbalanced by curcumin treatment in both types of cells.

\section{Discussion}

An increasing volume of evidence suggests that EMT plays a key role in cancer progression, cancer stem cell intravasation, establishment of metastasis and treatment resistance, resulting in a marked increase in disease aggressiveness and poorer disease outcome and overall patient survival (19). Pancreatic cancer, an aggressive and lethal malignant disease, is predicted to become the leading cause of cancer-related mortality in the USA by 2050 (20). The poor prognosis of pancreatic cancer patients is mainly attributed to the metastatic predilection of cancer cells. Although some tumors appear to be resectable, in reality surgery is not curative, due to the microscopic systemic spread that occurs prior to surgical resection (21). Our previous studies demonstrated that several factors may induce EMT in pancreatic cancer cells, thereby promoting tumor progression, including a hypoxic environment (8), a hyperglycemic environment (11), as well as the induction SOD (12). The aim of the present study was to determine whether curcumin has the potential to inhibit SOD-induced cell invasion and migration 

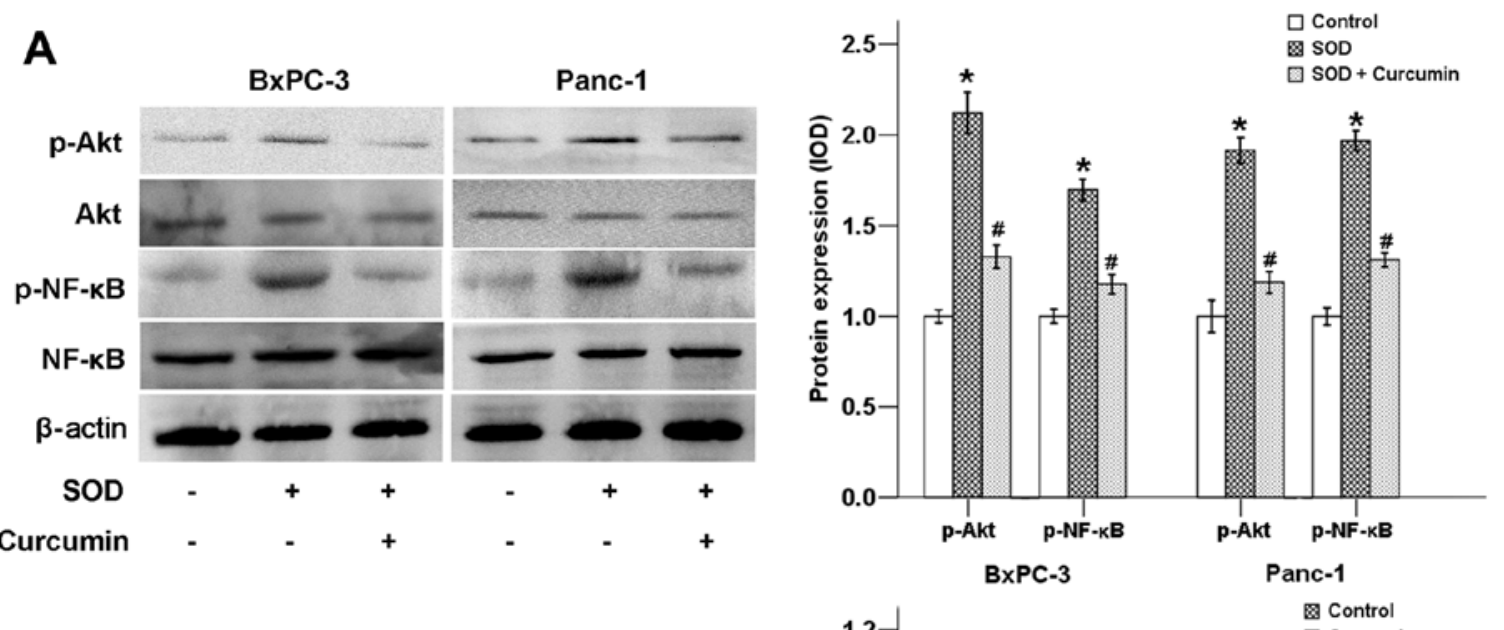

B
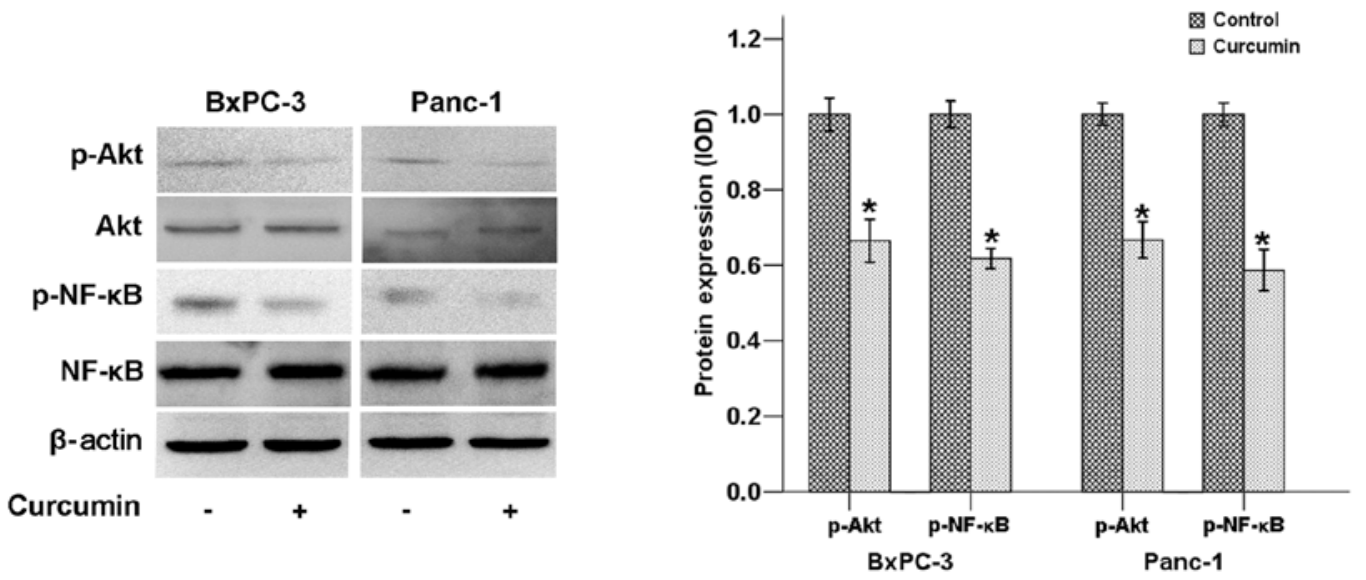

C
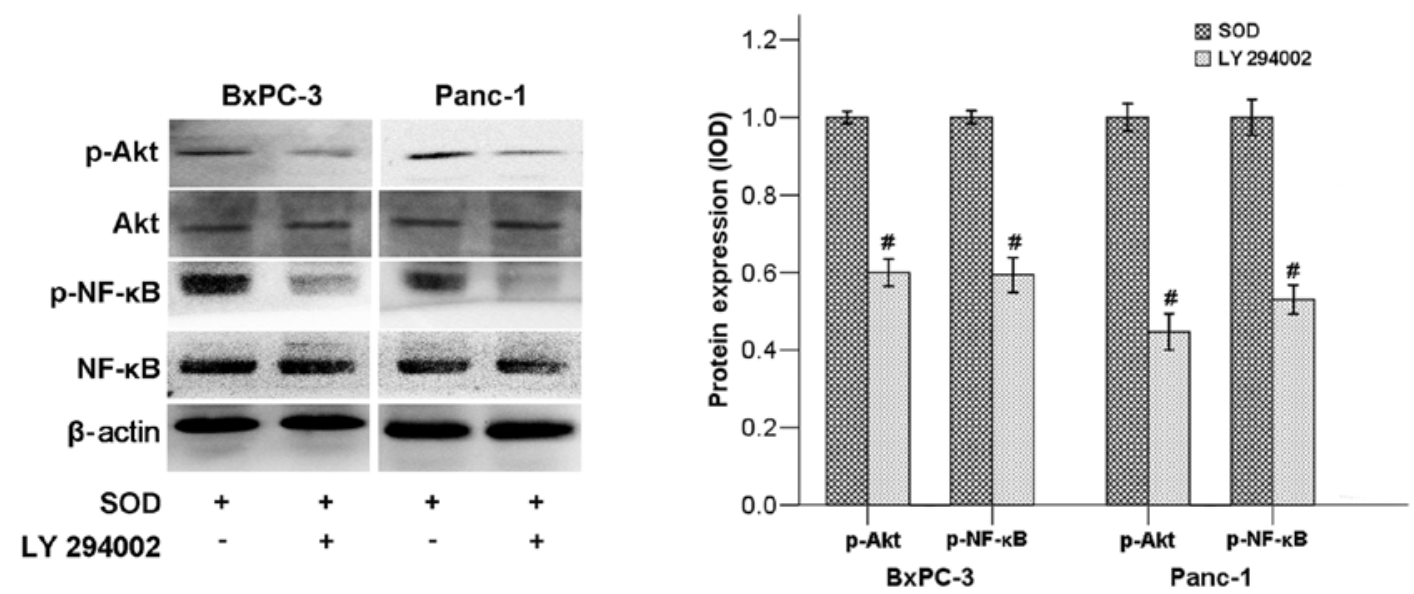

Figure 3. Roles of curcumin and LY 294002 in SOD-induced phosphorylation of Akt and NF-kB. (A) BxPC-3 and Panc-1 cells were pretreated with curcumin $(20 \mu \mathrm{M})$ and then exposed to SOD $(400 \mathrm{U} / \mathrm{ml})$ for $24 \mathrm{~h}$ to evaluate the phosphorylation levels of Akt and NF- $\mathrm{kB}$. (B) Curcumin alone inhibited Akt and NF- $\mathrm{kB}$ phosphorylation in BxPC-3 and Panc-1 cells. (C) Pancreatic cancer cells were treated with LY $294002(10 \mu \mathrm{M})$, a PI3K inhibitor, for $24 \mathrm{~h}$, and used for western

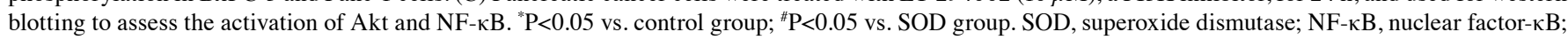
PI3K, phosphoinositide-3 kinase; IOD, integrated optical density.

and EMT in the BxPC-3 and Panc-1 cell lines, and elucidate the possible related mechanism.

Our findings demonstrated that curcumin significantly decreased SOD-induced production of ROS and $\mathrm{H}_{2} \mathrm{O}_{2}$ in pancreatic cancer cells. Curcumin, CAT (a scavenger of $\mathrm{H}_{2} \mathrm{O}_{2}$ ) and LY 294002 (a PI3K inhibitor) were able to suppress SOD-induced migration and invasion of BxPC-3 and Panc-1 cells. SOD-modulated cancer cell morphology and the expression of E-cadherin, N-cadherin and vimentin were markedly affected by curcumin. In addition, SOD-induced activation of the PI3K/Akt signaling pathway and the transcription factor $\mathrm{NF}-\kappa \mathrm{B}$ were also suppressed by curcumin. It is known that $\mathrm{H}_{2} \mathrm{O}_{2}$ induces ERK and Akt activation through a number of mechanisms. For example, Nrf2 is a transcription factor that plays a key role in controlling the response to oxidative stress by regulating antioxidant enzymes. The activation of Nrf2 may also be mediated by additional signal transduction pathways, such as ERK, AMPK or PI3K/Akt, exerting antioxidant effects, which mediate enhanced resistance to oxidative stress (22). The thioredoxin/thioredoxin reductase/TXNIP system is 

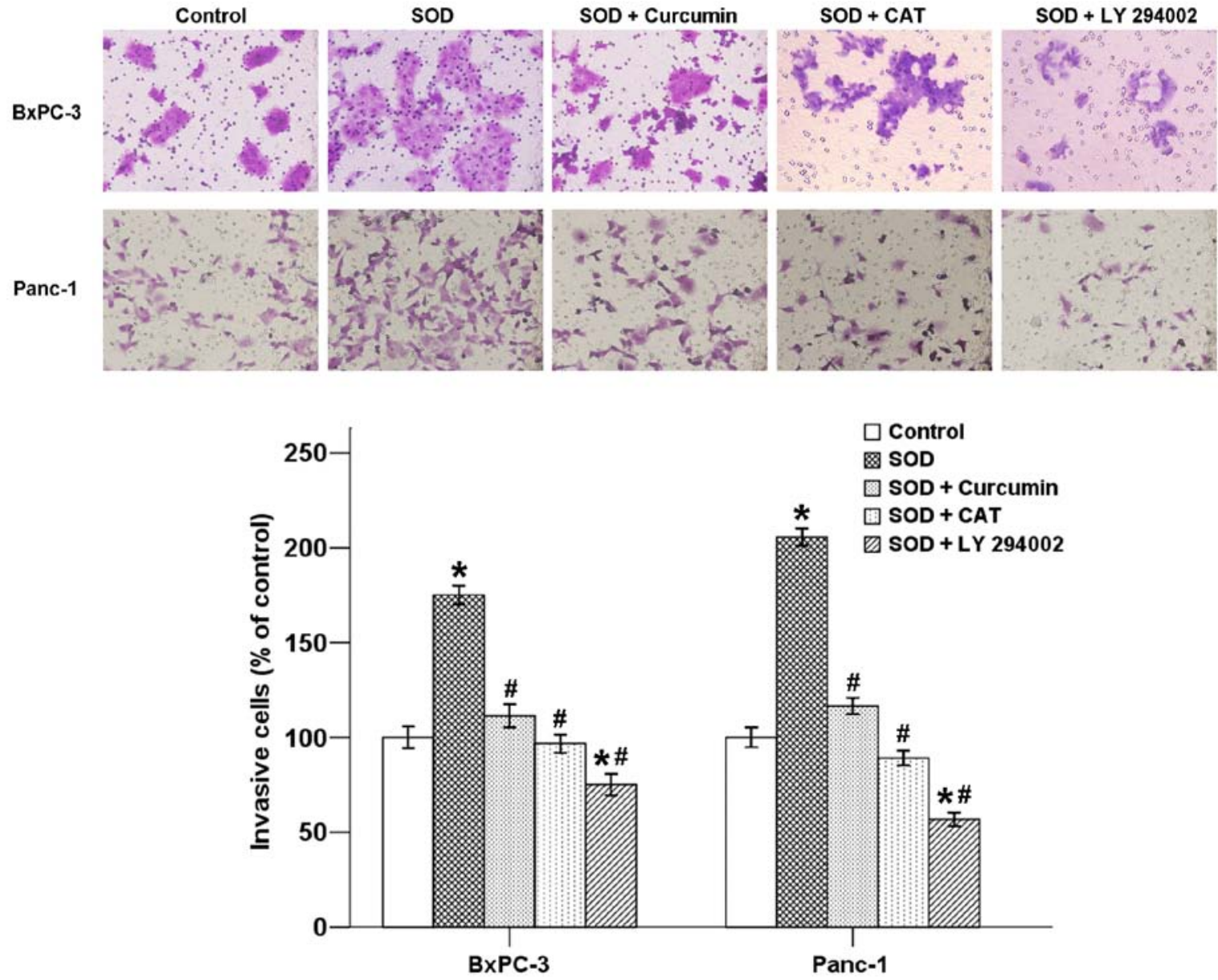

Figure 4. Effects of curcumin on SOD-induced invasive ability of pancreatic cancer cells. Pancreatic cancer cells were exposed to SOD (400 U/ml) as well as $20 \mu \mathrm{M}$ curcumin, $400 \mathrm{U} / \mathrm{ml} \mathrm{CAT}$, or $10 \mu \mathrm{M} \mathrm{LY} 294002$ for $48 \mathrm{~h}$. Cell invasion was observed and photographed, and the percentage of invading cells was calculated. The number of migrated cells was quantified by counting the number of cells from 10 random fields at a magnification of $\mathrm{x} 200$. ${ }^{*} \mathrm{P}<0.05$ vs. control group; ${ }^{\text {P }}<0.05$ vs. SOD group. SOD, superoxide dismutase; CAT, catalase.

also involved in oxidative stress and the ERK pathway, as it was previously proven that hyperglycemia-induced TXNIP expression is involved in diabetes-mediated oxidative stress in pancreatic cancer via the p38, MAPK and ERK pathways (23). Previous studies demonstrated that $\mathrm{H}_{2} \mathrm{O}_{2}$ contributes to both copper-zinc SOD (CuZnSOD)- and manganese SOD (MnSOD)-induced cancer progression through activation of Akt and ERK $(12,24,25)$, while curcumin suppresses $\mathrm{H}_{2} \mathrm{O}_{2}$-induced migration and invasion of pancreatic cancer cells (26). Our results revealed that curcumin suppresses SOD-induced EMT in pancreatic cancer, which may be associated with the $\mathrm{H}_{2} \mathrm{O}_{2} / \mathrm{Akt} / \mathrm{NF}-\kappa \mathrm{B}$ axis.

ROS and the activation of redox-sensitive signaling pathways are important participants in the development of neoplasms (27). SOD is a type of primary cellular antioxidant catalyzing the conversion of superoxide to $\mathrm{H}_{2} \mathrm{O}_{2}$, which has been proven to favor tumor progression (12). There are three members of the SOD family present in mammals, namely CuZnSOD, MnSOD and extracellular SOD (EC-SOD), which are located in different places inside or outside the cell (28). Epidemiological evidence has indicated that increased levels of SOD were observed in several tumor types along with tumor progression from early-stage (non-invasive) to late-stage (metastatic) (29). SOD polymorphism is associated with increased risk of prostate cancer, esophageal cancer, non-Hodgkin lymphoma, lung cancer and colorectal cancer $(30,31)$. The MnSOD-1221G>A AA genotype carriers exhibited a significantly increased risk of pancreatic cancer among those with a low dietary vitamin $\mathrm{E}$ intake (32). In addition, both in vitro and in vivo studies demonstrated that cancer cells containing elevated levels of SOD exhibit a propensity for metastasis, proliferation and resistance to apoptosis. Hart et al (33) revealed that SOD-induced $\mathrm{H}_{2} \mathrm{O}_{2}$ sustained the Warburg effect through AMPK-dependent signaling, enabling cancer cell survival. Chronic inflammation is a major activator of the metastatic cascade. Tumor-associated inflammation also participates in the regulation of EMT, which contributes to cancer invasion and metastasis. Yi et al (34) recently reported that SOD may favor inflammation-mediated EMT and migration of tumor cells in AFG1-induced lung adenocarcinoma. We have previously reported that SOD-dependent production of ROS promoted the invasion of pancreatic cancer cells (12). The present study demonstrated that curcumin effectively inhibited SOD-induced EMT in the BxPC-3 and Panc-1 pancreatic cancer cell lines.

Curcumin is a bioactive natural compound, which has been proven to restrain initiation, progression and metastasis of multifarious tumors, including pancreatic cancer (8). More importantly, curcumin is associated with minimal toxicity and it is safe at a high dose, as demonstrated by human clinical trials, 


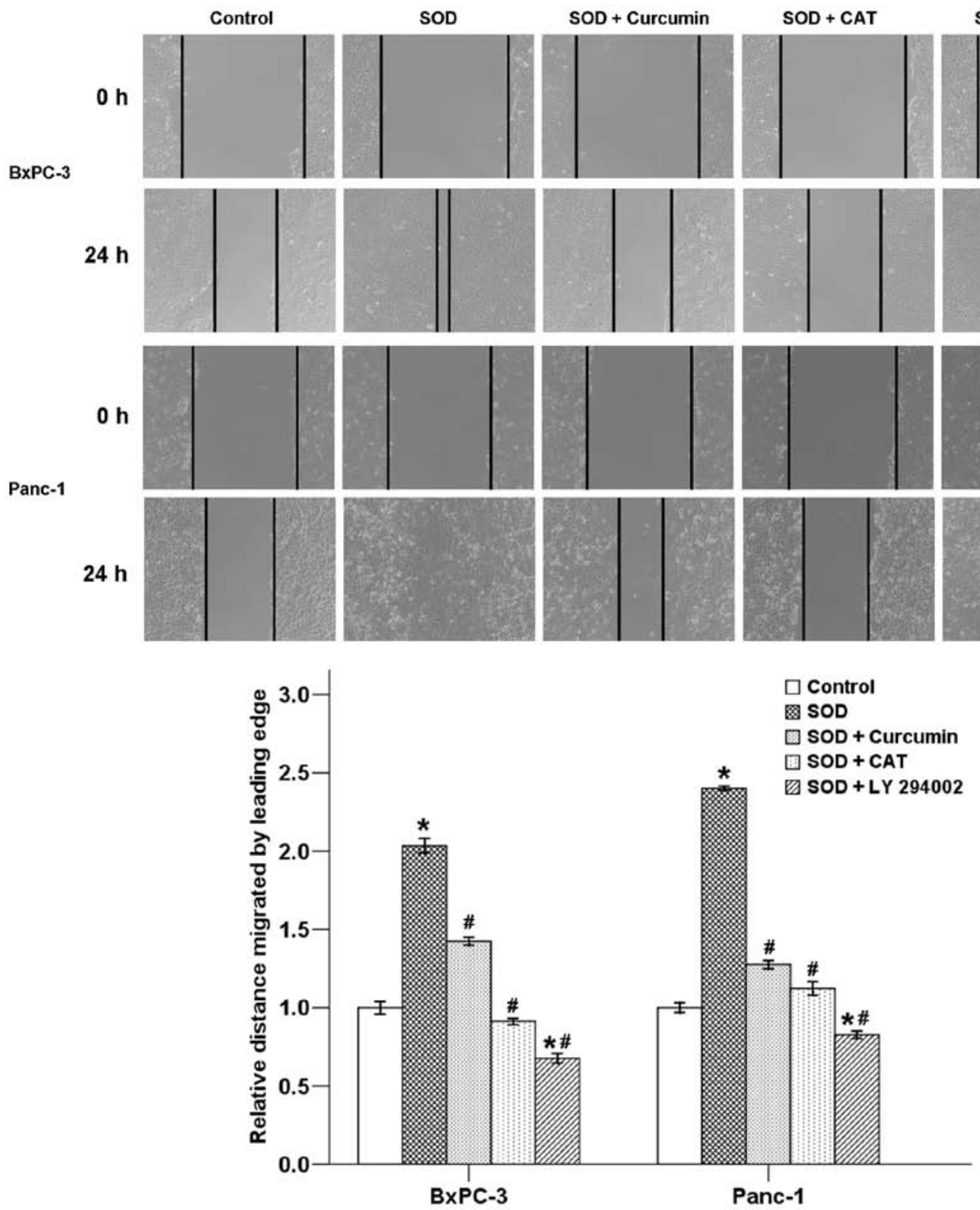

Figure 5. Effects of curcumin on SOD-induced migration of pancreatic cancer cells. The confluent monolayer was scratched with a sterile pipette tip, and the cancer cells were allowed to migrate for $24 \mathrm{~h}$. The migratory ability of both BxPC-3 and Panc-1 cells was enhanced by SOD, whereas the addition of $20 \mu \mathrm{M}$ curcumin, $400 \mathrm{U} / \mathrm{ml} \mathrm{CAT}$, or $10 \mu \mathrm{M}$ LY 294002 reduced cell migration. " $\mathrm{P}<0.05$ vs. control group; ${ }^{*} \mathrm{P}<0.05$ vs. SOD group. SOD, superoxide dismutase; CAT, catalase.

in contrast with conventional cytotoxic drugs (35). Curcumin exerts its anticancer effects via multiple signaling pathways, such as Notch, mammalian target of rapamycin (mTOR), MAPK and NF- $\mathrm{B}$ (5,36-39). Zhou et al (36) proved that curcumin suppressed pancreatic cancer cell growth, induced apoptosis and cell cycle arrest, weakened clonogenic potential, and inhibited migration and invasion via suppression of YAP/TAZ and Notch signaling. Treatment with curcumin also effectively attenuated tobacco smoke-induced activation of ERK and JNK MAPK pathways, AP-1 proteins and EMT alterations in mouse liver (5). Curcumin was also found to be able to induce autophagy and activate lysosomal function via its inhibitory effects on the Akt-mTOR signaling pathway and via direct targeting and activation of TFEB (37). In addition, curcumin exerted its anticancer effects both alone and in combination with other anticancer drugs. It has been proven that curcumin promotes the anticancer effects of gemcitabine via suppression of cancer cell proliferation, angiogenesis and inhibition of the $\mathrm{NF}-\kappa \mathrm{B}$ pathway in a pancreatic cancer model (38). A recent study indicated that co-treatment with metformin and curcumin not only induced apoptosis of hepatocellular carcinoma cells through activating the mitochondrial pathways, but also suppressed the invasion and metastasis of cancer cells and angiogenesis of human umbilical vein endothelial cells via suppression of PI3K/Akt/mTOR/NF- $\mathrm{BB}$ and EGFR/STAT3 signaling (39). Our recent study demonstrated that curcumin was able to suppress hypoxia-induced pancreatic cancer EMT and metastasis by inhibiting the hedgehog signaling pathway (8). It was also proven that curcumin was able to suppress cell migration and invasion through suppression of the ROS/ERK/NF- $\mathrm{B}$ signaling pathway (26). It was previously demonstrated that curcumin inhibited pancreatic 


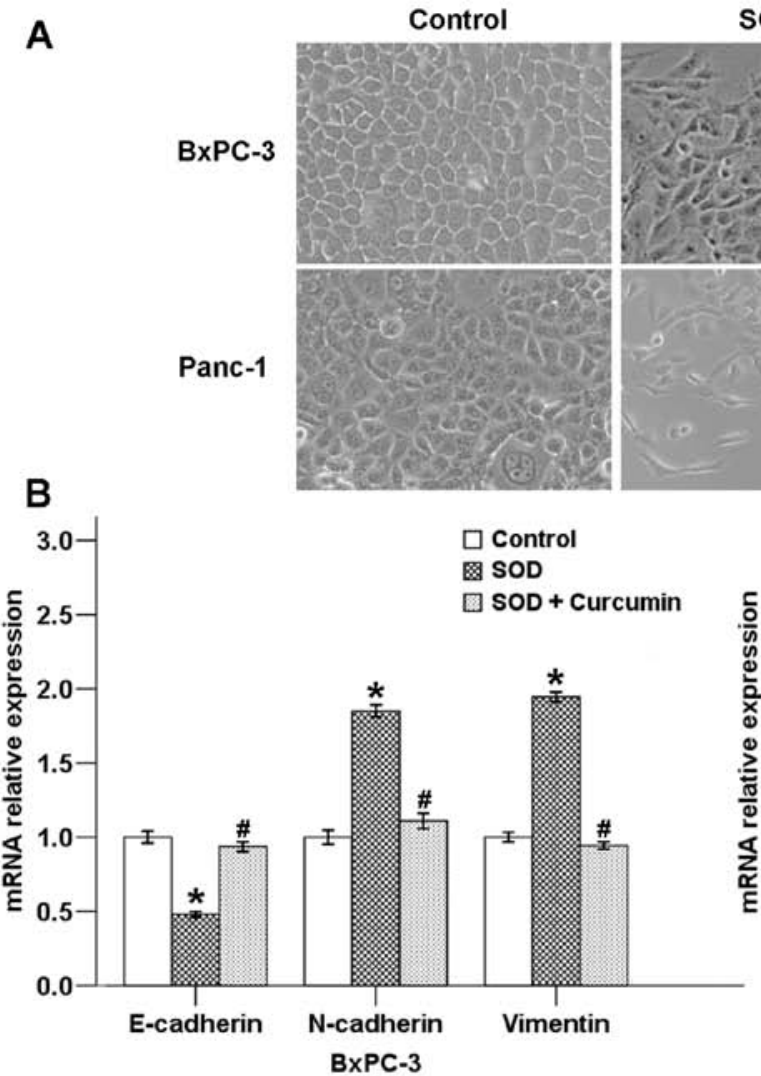

C
SOD

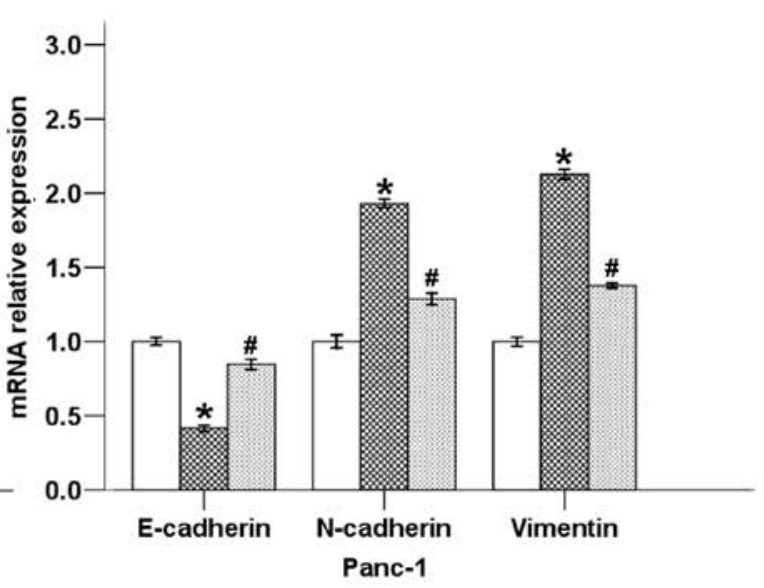

SOD + Curcumin

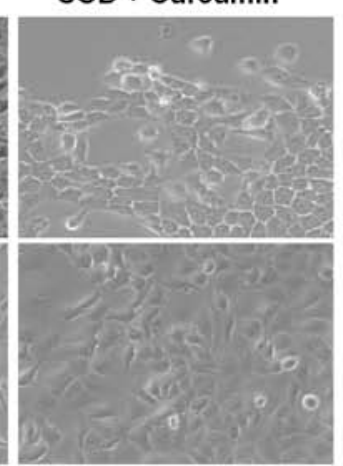

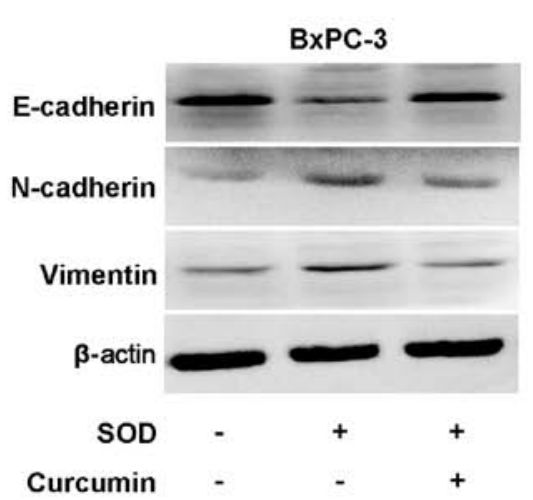

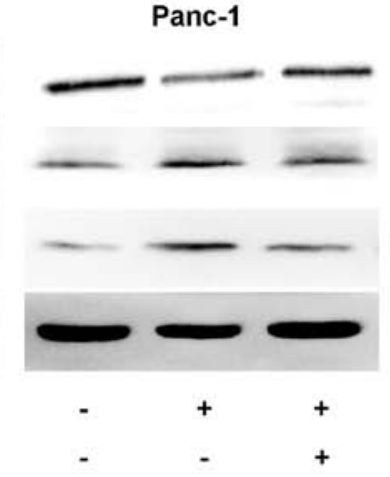

Figure 6. Effects of curcumin on SOD-induced EMT in pancreatic cancer cells. (A) Cells were treated with $400 \mathrm{U} / \mathrm{ml}$ SOD and the process of EMT was observed based on morphological changes of the cancer cells. Curcumin inhibited this effect of SOD. (B) Treatment with curcumin reduced the effects of SOD on the expression of E-cadherin, N-cadherin and vimentin at the mRNA level in BxPC-3 and Panc-1 cells. (C) Treatment with curcumin also decreased the effects of SOD on the expression of EMT-related genes at the protein level in pancreatic cancer cells. ${ }^{*} \mathrm{P}<0.05$ vs. control group; ${ }^{\#} \mathrm{P}<0.05$ vs. SOD group. EMT, epithelial-to-mesenchymal transition; SOD, superoxide dismutase.

cancer cell migration and invasion and suppressed NEDD4 expression (40). In our previous study, we also demonstrated that LY294002 exposure for $24 \mathrm{~h}$ reduced the migration of pancreatic cancer cells without the addition of SOD (41). The present study revealed that curcumin inhibited the effects of SOD in pancreatic cancer through suppression of the $\mathrm{PI} 3 \mathrm{~K} / \mathrm{Akt} / \mathrm{NF}-\kappa \mathrm{B}$ signaling pathway in pancreatic cancer cells.

The PI3K/Akt signaling pathway is one of the most frequently changing signaling networks in human cancer, and has long been identified as being implicated in cancer metastasis. Akt is hyperactivated in cancer cells through multiple mechanisms, including the loss of phosphatase and tensin homolog, mutations that activate the catalytic subunit of PI3K, mutations that activate Akt isoforms, and amplification of the genes encoding the catalytic subunit of PI3K and Akt (42).
Increased activation of PI3K/Akt signaling has been found in $\sim 50 \%$ of pancreatic cancers, which is usually associated with a low grade of tumor differentiation and is correlated with a poor prognosis. PI3K/Akt signaling modulates a series of cellular functions, including cell transformation, proliferation, growth, motility and survival (43). Our previous study demonstrated that resveratrol was highly efficient in inhibiting the proliferation, migration and invasion of pancreatic cancer cells in vitro by regulating EMT-related factors via the PI3K/Akt/NF- $\mathrm{B}$ signaling pathway (41). Recent studies demonstrated that both CuZnSOD and MnSOD can activate PI3K/Akt signaling in cancer cells $(24,25)$. The results of the present study revealed that curcumin has great therapeutic potential, as it can restrain SOD-induced activation of $\mathrm{p}-\mathrm{Akt}$ and $\mathrm{p}-\mathrm{NF}-\kappa \mathrm{B}$. Following suppression of the PI3K/Akt signaling pathway by LY 249002, 
the expression of $\mathrm{p}-\mathrm{Akt}$ and $\mathrm{NF}-\kappa \mathrm{B}$ decreased, and the invasive and migratory abilities of pancreatic cancer cells were weakened.

In conclusion, the present study demonstrated that curcumin inhibited SOD-induced invasion and migration of pancreatic cancer cells by regulating EMT-related factors via the PI3K/ $\mathrm{Akt} / \mathrm{NF}-\kappa \mathrm{B}$ signaling pathway. Therefore, inhibition of the $\mathrm{H}_{2} \mathrm{O}_{2} / \mathrm{Akt} / \mathrm{NF}-\kappa \mathrm{B}$ axis by curcumin may represent a promising option for the treatment of patients with pancreatic cancer.

\section{Acknowledgements}

Not applicable.

\section{Funding}

This study was supported by the Natural Science Basic Research Project of Shaanxi Province (grant no. 2017JM8037).

\section{Availability of data and materials}

The analysed data sets generated during the study are available from the corresponding authors on reasonable request.

\section{Author contributions}

QM and LC conceived the study; WL, ZWu and LC designed the study; WL, ZJ and XX conducted the experiments; and ZWa and LC performed the data analysis. All authors have read and approved this manuscript.

\section{Ethics approval and consent to participate}

Not applicable.

\section{Consent for publication}

Not applicable.

\section{Competing interests}

The authors declare that they have no competing interests.

\section{References}

1. Siegel RL, Miller KD and Jemal A: Cancer Statistics, 2017. CA Cancer J Clin 67: 7-30, 2017.

2. Lin QJ, Yang F, Jin C and Fu DL: Current status and progress of pancreatic cancer in China. World J Gastroenterol 21: 7988-8003, 2015.

3. Vincent A, Herman J, Schulick R, Hruban RH and Goggins M: Pancreatic cancer. Lancet 378: 607-620, 2011.

4. Shanmugam MK, Rane G, Kanchi MM, Arfuso F, Chinnathambi A, Zayed ME, Alharbi SA, Tan BK, Kumar AP and Sethi G: The multifaceted role of curcumin in cancer prevention and treatment. Molecules 20: 2728-2769, 2015 .

5. Liang Z, Wu R, Xie W, Xie C, Wu J, Geng S, Li X, Zhu M, Zhu W Zhu J, et al: Effects of curcumin on tobacco smoke-induced hepatic MAPK pathway activation and epithelial-mesenchymal transition in vivo. Phytother Res 31: 1230-1239, 2017.

6. Fu H, Wang C, Yang D, Wei Z, Xu J, Hu Z, Zhang Y, Wang W, Yan R and Cai Q: Curcumin regulates proliferation, autophagy, and apoptosis in gastric cancer cells by affecting PI3K and P53 signaling. J Cell Physiol: Sep 19, 2017 (Epub ahead of print) doi: $10.1002 /$ jep. 26190 .
7. Liang Z, Lu L, Mao J,Li X, Qian H and Xu W: Curcumin reversed chronic tobacco smoke exposure induced urocystic EMT and acquisition of cancer stem cells properties via Wnt $/ \beta$-catenin. Cell Death Dis 8: e3066, 2017.

8. Cao L, Xiao X, Lei J, Duan W, Ma Q and Li W: Curcumin inhibits hypoxia-induced epithelial mesenchymal transition in pancreatic cancer cells via suppression of the hedgehog signaling pathway. Oncol Rep 35: 3728-3734, 2016.

9. Bhatia S, Monkman J, Toh AKL, Nagaraj SH and Thompson EW Targeting epithelial-mesenchymal plasticity in cancer: Clinical and preclinical advances in therapy and monitoring. Biochem J 474: 3269-3306, 2017.

10. Li W, Ma Q, Liu J, Han L, Ma G, Liu H, Shan T, Xie K and Wu E: Hyperglycemia as a mechanism of pancreatic cancer metastasis. Front Biosci (Landmark Ed) 17: 1761-1774, 2012.

11. Li W,Zhang L, Chen X, Jiang Z,Zong L and Ma Q: Hyperglycemia promotes the epithelial-mesenchymal transition of pancreatic cancer via hydrogen peroxide. Oxid Med Cell Longev 2016: 5190314, 2016.

12. Li W, Cao L, Han L, Xu Q and Ma Q: Superoxide dismutase promotes the epithelial-mesenchymal transition of pancreatic cancer cells via activation of the $\mathrm{H}_{2} \mathrm{O}_{2} / E R K / N F-\kappa B$ axis. Int $\mathrm{J}$ Oncol 46: 2613-2620, 2015.

13. Costa A, Scholer-Dahirel A and Mechta-Grigoriou F: The role of reactive oxygen species and metabolism on cancer cells and their microenvironment. Semin Cancer Biol 25: 23-32, 2014.

14. Guo L, Tan K, Wang H and Zhang X: Pterostilbene inhibits hepatocellular carcinoma through p53/SOD2/ROS-mediated mitochondrial apoptosis. Oncol Rep 36: 3233-3240, 2016.

15. Livak KJ and Schmittgen TD: Analysis of relative gene expression data using real-time quantitative PCR and the 2(-Delta Delta $\mathrm{C}(\mathrm{T})$ ) method. Methods 25: 402-408, 2001.

16. Martinez-Useros J, Li W, Cabeza-Morales M and GarciaFoncillas J: Oxidative stress: A new target for pancreatic cancer prognosis and treatment. J Clin Med 6: E29, 2017.

17. Mayer IA and Arteaga CL: The PI3K/AKT pathway as a target for cancer treatment. Annu Rev Med 67: 11-28, 2016.

18. Yu X, Wu Q, Wang L, Zhao Y, Zhang Q, Meng Q, Pawan and Wang S: Silencing of ST6GalNAc I suppresses the proliferation, migration and invasion of hepatocarcinoma cells through PI3K/AKT/NF-kB pathway. Tumour Biol 37: 12213-12221, 2016.

19. Gaianigo N, Melisi D and Carbone C: EMT and treatment resistance in pancreatic cancer. Cancers (Basel) 9: 9, 2017.

20. Jemal A, Bray F, Center MM, Ferlay J, Ward E and Forman D: Global cancer statistics. CA Cancer J Clin 61: 69-90, 2011.

21. Castellanos EH, Cardin DB and Berlin JD: Treatment of earlystage pancreatic cancer. Oncology (Williston Park) 25: 182-189, 2011.

22. Claudia L, Jette R, Rudolf L, Ludger AW, and Barbara Sr: Hydrogen peroxide - production, fate and role in redox signaling of tumor cells. Cell Commun Signal 13: 39, 2015.

23. Li W, Wu Z, Ma Q, Liu J, Xu Q, Han L, Duan W, Lv Y, Wang F, Reindl KM, et al: Hyperglycemia regulates TXNIP/TRX/ROS axis via p38 MAPK and ERK pathways in pancreatic cancer. Curr Cancer Drug Targets 14: 348-356, 2014

24. Li F, Wang H, Huang C, Lin J, Zhu G, Hu R and Feng H: Hydrogen peroxide contributes to the manganese superoxide dismutase promotion of migration and invasion in glioma cells. Free Radic Res 45: 1154-1161, 2011.

25. Damiano S, Petrozziello T, Ucci V, Amente S, Santillo M and Mondola P: $\mathrm{Cu}-\mathrm{Zn}$ superoxide dismutase activates muscarinic acetylcholine M1 receptor pathway in neuroblastoma cells. Mol Cell Neurosci 52: 31-37, 2013.

26. Cao L, Liu J, Zhang L, Xiao X and Li W: Curcumin inhibits $\mathrm{H}_{2} \mathrm{O}_{2}$-induced invasion and migration of human pancreatic cancer via suppression of the ERK/NF- $\kappa \mathrm{B}$ pathway. Oncol Rep 36: 2245-2251, 2016.

27. Schieber M and Chandel NS: ROS function in redox signaling and oxidative stress. Curr Biol 24: R453-R462, 2014.

28. Griess B, Tom E, Domann F and Teoh-Fitzgerald M: Extracellular superoxide dismutase and its role in cancer. Free Radic Biol Med 112: 464-479, 2017.

29. Hempel N, Carrico PM and Melendez JA: Manganese superoxide dismutase (Sod2) and redox-control of signaling events that drive metastasis. Anticancer Agents Med Chem 11: 191-201, 2011.

30. Sun GG, Wang YD, Lu YF and Hu WN: Different association of manganese superoxide dismutase gene polymorphisms with risk of prostate, esophageal, and lung cancers: Evidence from a meta-analysis of 20,025 subjects. Asian Pac J Cancer Prev 14: $1937-1943,2013$ 
31. Kang SW: Superoxide dismutase 2 gene and cancer risk: Evidence from an updated meta-analysis. Int J Clin Exp Med 8: 14647-14655, 2015.

32. Tang H, Dong X, Day RS, Hassan MM and Li D: Antioxidant genes, diabetes and dietary antioxidants in association with risk of pancreatic cancer. Carcinogenesis 31: 607-613, 2010.

33. Hart PC, Mao M, de Abreu AL, Ansenberger-Fricano K, Ekoue DN, Ganini D, Kajdacsy-Balla A, Diamond AM, Minshall RD, Consolaro ME, et al: MnSOD upregulation sustains the Warburg effect via mitochondrial ROS and AMPK-dependent signalling in cancer. Nat Commun 6: 6053, 2015.

34. Yi L, Shen H, Zhao M, Shao P, Liu C, Cui J, Wang J, Wang C, Guo N, Kang L, et al: Inflammation-mediated SOD-2 upregulation contributes to epithelial-mesenchymal transition and migration of tumor cells in aflatoxin G1-induced lung adenocarcinoma. Sci Rep 7: 7953, 2017.

35. Yang C, Su X, Liu A, Zhang L, Yu A, Xi Y and Zhai G: Advances in clinical study of curcumin. Curr Pharm Des 19: 1966-1973, 2013.

36. Zhou X, Su J, Feng S, Wang L, Yin X, Yan J and Wang Z: Antitumor activity of curcumin is involved in down-regulation of YAP/TAZ expression in pancreatic cancer cells. Oncotarget 7: 79076-79088, 2016.

37. Zhang J, Wang J, Xu J, Lu Y, Jiang J, Wang L, Shen HM and Xia D: Curcumin targets the TFEB-lysosome pathway for induction of autophagy. Oncotarget 7: 75659-75671, 2016.
38. Kunnumakkara AB, Guha S, Krishnan S, Diagaradjane P, Gelovani J and Aggarwal BB: Curcumin potentiates antitumor activity of gemcitabine in an orthotopic model of pancreatic cancer through suppression of proliferation, angiogenesis, and inhibition of nuclear factor-kappaB-regulated gene products. Cancer Res 67: 3853-3861, 2007.

39. Zhang HH, Zhang Y, Cheng YN, Gong FL, Cao ZQ, Yu LG and Guo XL: Metformin incombination with curcumin inhibits the growth, metastasis, and angiogenesis of hepatocellular carcinoma in vitro and in vivo. Mol Carcinog 57: 44-56, 2018.

40. Su J, Zhou X, Yin X, Wang L, Zhao Z, Hou Y, Zheng N, Xia J and Wang $Z$ : The effects of curcumin on proliferation, apoptosis, invasion, and NEDD4 expression in pancreatic cancer. Biochem Pharmacol 140: 28-40, 2017.

41. Li W, Ma J, Ma Q, Li B, Han L, Liu J, Xu Q, Duan W, Yu S, Wang F, et al: Resveratrol inhibits the epithelial-mesenchymal transition of pancreatic cancer cells via suppression of the PI-3K/Akt/NF- $\mathrm{KB}$ pathway. Curr Med Chem 20: 4185-4194, 2013.

42. Wang Q, Chen X and Hay N: Akt as a target for cancer therapy: More is not always better (lessons from studies in mice). $\mathrm{Br} J$ Cancer 117: 159-163, 2017.

43. Baer R, Cintas C, Therville N and Guillermet-Guibert J: Implication of PI3K/Akt pathway in pancreatic cancer: When PI3K isoforms matter? Adv Biol Regul 59: 19-35, 2015. 